Urol. Prax. $2021 \cdot$ 23:144-150

https://doi.org/10.1007/s41973-021-00155-w

Angenommen: 6. Oktober 2021

Online publiziert: 22. November 2021

(c) Der/die Autor(en) 2021

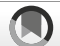

\section{Einleitung}

Definitionsgemäss wird erst $a b$ dem 5. Lebensjahr von einer Enuresis nocturna gesprochen, vorher entspricht das nächtliche Einnässen im Kindesalter einem physiologischen Reifungsprozess [1]. Die Prävalenz ist altersabhängig und nimmt mit steigendem Alter deutlich ab. Bei 5-jährigen Kindern sind etwa $15 \%$ betroffen, bei 10-Jährigen weniger als $5 \%$, und bei über 15 -jährigen Jugendlichen sind 98-99\% nachtsüber kontinent [2-4]. Knaben sind häufiger betroffen als Mädchen, die jährliche Spontanheilungsrate beträgt etwa $15 \%$ $[5,6]$.

Gemäss der Terminologie der ICCS („International Children's Continence Society") sprechen wir von einer monosymptomatischen Enuresis nocturna beim Kind, wenn ausser der nächtlichen Symptomatik keine weitere Blasenentleerungsstörung tagsüber und/ oder Grunderkrankungen vorhanden sind [1].

Die Abb. 1 zeigt schematisch die Einteilung der Formen der kindlichen Enuresis nocturna, welche der internationalen Beschreibung entsprechen. Handelt es sich um eine sekundäre Enuresis nocturna, war das Kind vorher mindestens 6 Monate am Stück komplett trocken [1].

Eine nichtmonosymptomatische Enuresis nocturna geht definitionsgemäss mit weiteren Symptomen des unteren Harntraktes (LUTS [„lower urinary tract symptoms"]) einher, wie z. B. einer Urgesymptomatik, Rückhaltemanövern, Inkontinenz tagsüber usw. In etwa $20 \%$ der Fälle von Kindern mit nächtlicher Einnässsymptomatik zeigt sich auch tagsüber eine Blasenentleerungsstörung,

\title{
Enuresis nocturna im Kindes- und Jugendalter
}

und etwa $15 \%$ der betroffenen Kinder zeigen Defäkationsstörungen im Sinn einer „bladder bowel dysfunktion“(BBD; [7]).

Diese Unterscheidung ist letztlich wichtig zur Festlegung der weiteren Therapie. Es ist meistens nicht zielführend, die nächtliche Symptomatik zu behandeln, die grundliegende Blasenentleerungsstörung und/oder Defäkationsstörung aber ausser Acht zu lassen. In der Praxis trifft man immer wieder auf hartnäckige Fälle. Diese Kinder haben schon sämtliche medikamentöse Therapien und nervenaufreibende Behandlungsversuche mit dem Weckapparat hinter sich, eine geeignete Therapie für die zugrunde liegende Blasenentleerungsstörung und/oder Obstipation wurde aber nicht etabliert. Etwas salopp gesagt, wird es in der Nacht nicht besser, solange es am Tag nicht funktioniert.

\section{Pathogenese}

Der genaue Mechanismus des normalen Reifungsprozesses der Blasenfunktion und entsprechende Störungen sind zum aktuellen Zeitpunkt noch nicht im Detail bekannt. Letztlich ist es ein komplexes Zusammenspiel von peripheren Nerven, spinalen und zentralen Arealen, welches bei hohem Auslasswiderstand eine Urinspeicherung in einem Niederdrucksystem ermöglicht. Bei der Urinentleerung senkt sich der Auslasswiederstand und die Detrusormuskulatur wird kontrahiert. Im Säuglingsalter ist die Blasenentleerung suffizient (trotz noch physiologischem Restharn), aber unkoordiniert und findet am ehesten über die Blasenfüllung statt. Die Blasenentleerung kann auch infolge eines neurologischen Stimulus in Gang kom- men (Windel wechseln, Kitzeln, Baden usw., [8]).

Im Kleinkindesalter erfolgt die zunehmende Reifung der Blasenkontrolle. Im ersten Schritt kann das Kind die Blasenfüllung wahrnehmen. Im weiteren Verlauf lernt es, die Detrusorkontraktionen aktiv zu unterdrücken, und ab dem Alter von etwa 5 Jahren können die Kinder Sphinkter und Detrusor gut miteinander koordinieren. Die nächtliche Blasenkontrolle wird dann Monate bis Jahre später erreicht [9].

Die Ursachen für ein nächtliches Einnässen sind vielfältig, aber psychologische/psychiatrische Gründe sind dabei eher selten und eher Folge als Ursache der Enuresis nocturna. Über eine genetische Prädisposition wird berichtet. Die Konkordanz bei monozygoten Zwillingen ist im Vergleich zu dizygoten Zwillingen etwa doppelt so hoch (68\% vs. $36 \%,[10])$.

Falls ein Elternteil ebenfalls eine Enuresis nocturna hatte, sind etwa 50\% der Kinder davon auch betroffen. War diese bei beiden Elternteilen vorhanden, steigt die Rate auf $75 \%$ [11].

Da die meisten Fälle von monosymptomatischer Enuresis nocturna eine sehr hohe Spontanheilungsrate zeigen, liegt der Schluss nahe, dass es sich um eine Reifungsverzögerung der Blasenentleerung handelt.

Eine gesteigerte nächtliche Urinproduktion kann durchaus im Rahmen einer mehrheitlichen abendlichen Flüssigkeitszufuhr verursacht sein (wer hat schon Zeit, tagsüber in der Schulpause zu trinken?). Von einer nächtlichen Polyurie wird gesprochen, wenn die nächtliche Urinproduktion $>130 \%$ der zu erwartenden Blasenkapazität entspricht. 


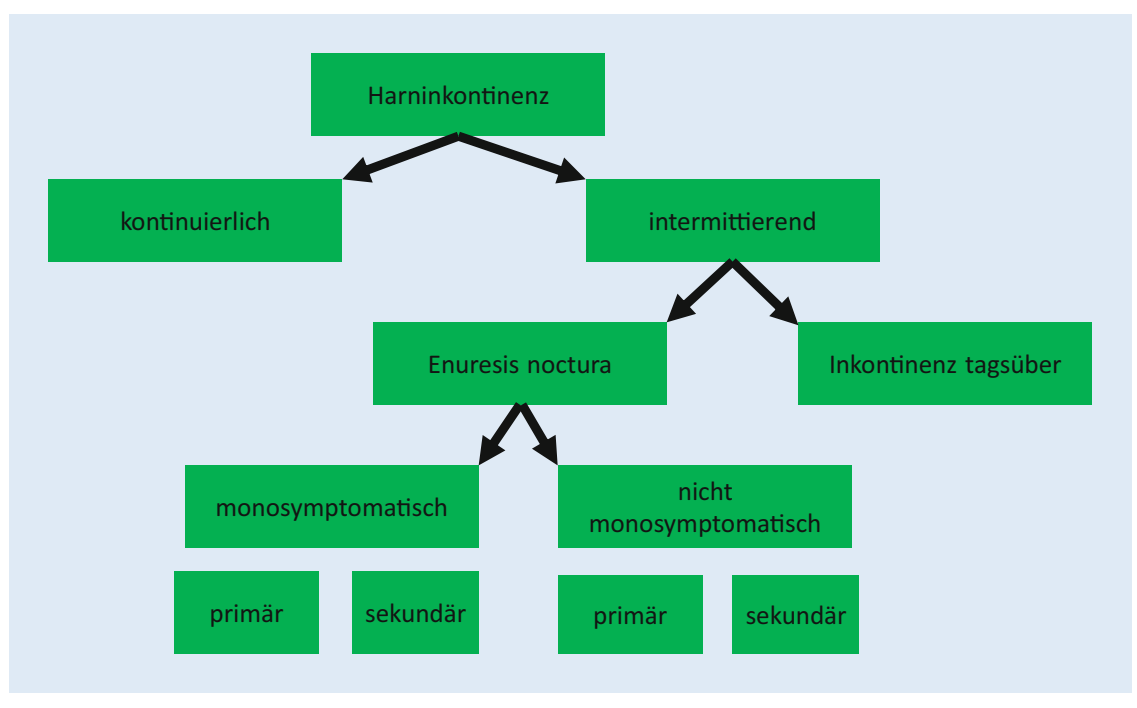

Abb. $1 \Delta$ Einteilung der Enuresis nocturna gemäss ICCS(„International Children's Continence Society")

Zusätzlich werden eine verminderte Sensibilität auf das antidiuretische Hormon $(\mathrm{ADH})$ und/oder eine verminderte Sekretion von $\mathrm{ADH}$ postuliert. Jedoch ist dies keine Erklärung, warum die Kinder nachts nicht aufwachen. Die Rolle der inadäquaten $\mathrm{ADH}$-Sekretion/-Sensibilität ist aber weiterhin unklar, und bisher gibt es keinen verlässlichen laborchemischen Parameter, welcher das Ansprechen auf eine Therapie mit einem ADHAnalogon quantifizieren könnte [12].

Eine verminderte Blasenkapazität (meistens funktionell bedingt) und ein hyperaktiver Detrusor mit oder ohne BBD sind durchaus weitere mögliche Ursachen für nächtliches Einnässen. In aller Regel zeigen die betroffenen Kinder aber auch tagsüber Symptome, sodass hier eher eine nichtmonosymptomatische Enuresis vorliegt. Selten kann eine isolierte nächtliche Detrusorhyperaktivität (bei gestörter zirkadianer Aktivierung der Beckenbodenmuskulatur) eine therapierefraktäre monosymptomatische Enuresis verursachen [13].

Anamnestisch wird fast immer von einem sehr tiefen Schlaf berichtet, wobei die Patienten kaum weckbar seien. Der Einfluss des Schlafmusters wird kontrovers diskutiert. Es gibt Studien, die eher tiefere Schlafepisoden während des Einnässereignisses aufzeigen [14], während andere eher einen oberflächlichen Schlaf dokumentieren [15] und wieder ande- re keinen signifikanten Unterschied zwischen den Gruppen finden [16].

Möglicherweise entsteht der Eindruck des tiefen Schlafes einfach dadurch, dass Eltern ihre Kinder nachts häufiger zu wecken versuchen. Wer weckt sonst schon ein Kind ohne nächtliche Symptome?

Harnwegsinfektionen, posteriore Urethralklappen bei Jungen, ein ektoper Ureter, chronische Nierenerkrankungen, Diabetes mellitus, Diabetes insipidus usw. können eine Enuresis auslösen und sollten ausgeschlossen werden. Des Weiteren gibt es vielfältige pädiatrische Grunderkrankungen, welche mit einem nächtlichen Einnässen einhergehen, wie z.B. eine Entwicklungsretardierung, neurologische Grunderkrankungen sowie das Aufmerksamkeitsdefizit- und Hyperaktivitätssyndrom (ADHS).

\section{"Workup"}

Zur besseren Differenzierung der Problematik des nächtlichen Einnässens sind eine gründliche Anamnese, die körperliche Untersuchung, eine Urinanalyse und, je nach Bedarf, eine apparative oder bildgebende Diagnostik notwendig [17].

Neben einer allgemeinpädiatrischen Anamnese wird das Augenmerk auf urologische Symptome gelegt. Folgende Parameter sind wichtig: Miktionsfrequenz und -volumen (gibt es tagsüber genügend Gelegenheiten und Zeit zum Toilettengang? Nimmt sich das Kind auf der Toilette genügend Zeit zur kompletten Entleerung?), Strahlstärke, Hinweise auf LUTS (Urgesymptomatik, Rückhaltemanöver, Inkontinenz tagsüber, unterbrochene Miktion), Harnwegsinfektionen und deren Häufigkeit, uronephrologische Familienanamnese [17].

Wie sieht die Flüssigkeitsaufnahme aus? Trinkt das Kind genügend? Hat es regelmässig Gelegenheit zum Trinken? Darf es während dem Unterricht trinken? Trinkt es eher abends? Häufig wird anamnestisch die Trinkmenge überschätzt („mein Kind trinkt genug“). Empfohlen sind im Kindergarten/Primarschulalter 6-8 Gläser pro Tag, dies entspricht $1,2-1,51$ pro Tag. Bei älteren Kindern wird eine Trinkmenge von 1,5-21 pro Tag empfohlen [17].

Zentral ist auch die Stuhlentleerung. Hier muss meistens spezifischer nachgefragt werden (Stuhlfrequenz, rezidivierende Bauchschmerzen, Enkopresis), da die meisten Menschen die Frage: „Sind Sieverstopft" mit Nein beantworten. Hilfreich ist hier die Bristol-Stuhlskala [17]. Durchfall ist im Kindesalter nicht selten ein Hinweis auf eine Obstipation. Da der harte Stuhl lange im Rektum verweilt, zersetzt sich der darüber liegende alte Stuhl mit der Zeit mikrobiologisch und wandert in flüssiger Form entlang der harten Stuhlballen im Rektum nach distal.

Zur Abklärung gehört ein Trink- und Miktionsprotokoll über 2-3 Tage. Dieses dient der Objektivierung von Miktionsfrequenz, Miktionsvolumen (Blasenkapazität), LUTS und Trinkverhalten. Ein Stuhlprotokoll ist ebenfalls notwendig [17].

Der klinische Status eines Kindes mit einer primären monosymptomatischen Enuresis nocturna ist in aller Regel unauffällig. Es zeigen sich ein normales perzentilengerechtes Wachstum und eine normale körperliche und geistige Entwicklung. Stuhlwalzen, meistens im linken Hemiabdomen, können häufig bei Obstipation palpiert werden. Der Rücken wird zur Detektion allfälliger spinaler Dysraphien lumbosakral inspiziert [17].

Das Genitale sollte in ruhiger Atmosphäre und, wenn immer möglich, im Beisein der Eltern untersucht werden. Dabei kann auch ein Blick auf die Un- 
Zusammenfassung · Résumé

Urol. Prax. 2021 23:144-150 https://doi.org/10.1007/s41973-021-00155-w

(c) Der/die Autor(en) 2021

\section{Sommer $\cdot$ S. Holland-Cunz $\cdot$ M. Frech}

\section{Enuresis nocturna im Kindes- und Jugendalter}

\section{Zusammenfassung}

Das nächtliche Einnässen im Kindesalter ist ein häufiger Konsultationsgrund im kinderurologischen Alltag. Nach heutiger Ansicht sind psychische Probleme („die Seele weint") meistens nicht ursächlich. Die Ätiologie ist multifaktoriell inklusive einer genetischen Prädisposition und bisher nicht im Detail geklärt. Aber für viele Familien ist dies eine belastende Situation, und sie kann das Selbstwertgefühl und psychische Wohlbefinden der Kinder und Jugendlichen deutlich beeinträchtigen.

Mit einer gründlichen kinderurologischen Anamnese, einem Trink- und Miktionsprotoll und körperlicher Untersuchung lassen sich kindliche Miktionsstörungen meistens gut differenzieren. Je nach Befund sind im
Verlauf weitere Untersuchungen indiziert, insbesondere wenn eine organische Ursache vermutet wird. Wichtig hierbei ist, zu unterscheiden, ob es sich um eine isolierte monosymptomatische Enuresis nocturna handelt oder die Enuresis nocturna Ausdruck einer weiteren kindlichen Miktionsstörung ist (nichtmonosymptomatische Enuresis nocturna).

Die Therapie richtet sich nach den möglichen Ursachen und dem Wunsch der Patienten und deren Familien. Eine monosymptomatische Enuresis nocturna hat durchaus einen sehr hohen Grad an Selbstheilungstendenz ohne Therapie oder nur mittels einfacher Verhaltensmassnahmen. Bei Therapiewunsch stehen eine medikamentöse Behandlung und/oder eine Konditionierung durch einen Weckapparat zur Verfügung.

Sofern es sich um eine nichtmonosymptomatische Enuresis nocturna handelt, sind o.g. Therapien meistens frustran. Der therapeutische Ansatz hierbei ist je nach Ursache individuell und reicht von einfachen Verhaltensmassnahmen (z. B. bei Reifungsverzögerung der Blasenentwicklung) bis hin zu operativen Verfahren (z. B. bei posterioren Urethralklappen beim Knaben).

\section{Schlüsselwörter}

Inkontinenz · Kindliche Miktionsstörung . Bettnässen · Weckapparat · Antidiuretisches Hormon

\section{Énurésie nocturne dans l'enfance et l'adolescence}

\section{Résumé}

L'énurésie nocturne dans l'enfance est une raison fréquente de consultation en urologie pédiatrique. On estime aujourd'hui que ce problème n'est que rarement dû à des problèmes psycho-affectifs («l'âme pleure»). L'étiologie est multifactorielle, y compris une prédisposition génétique, et n'est pas encore éclaircie dans les détails. Mais pour de nombreuses familles, il s'agit d'une situation pénible et susceptible de fortement affecter l'estime de soi et le bien-être mental des enfants et adolescents.

Les troubles mictionnels de l'enfant sont généralement bien différenciables par une anamnèse uro-pédiatrique approfondie, par un journal de bord sur l'apport liquidien et les mictions et par l'examen clinique. Selon les constats, d'autres examens peuvent encore être indiqués, surtout si une cause organique est suspectée. Il est important de distinguer s'il s'agit d'une énurésie nocturne monosymptomatique isolée ou si l'énurésie nocturne est la manifestation d'un autre trouble mictionnel de l'enfant (énurésie nocturne non monosymptomatique).

Le traitement dépendra des causes possibles et des préférences du patient et de sa famille. Une énurésie nocturne monosymptomatique présente fondamentalement une forte tendance à l'autoguérison sans aucun traitement ou à l'aide de simples habitudes à prendre. Si un traitement est souhaité, on peut recourir à une pharmacothérapie et/ou à un conditionnement à l'aide d'un appareil d'alarme.
Dans le cas d'une énurésie nocturne non monosymptomatique, les traitements susmentionnés sont généralement décevants. Ici, I'approche thérapeutique doit être définie au cas par cas selon la cause du problème; le spectre des options s'étend de simples mesures comportementales ( $p$. ex. lors d'un retard de maturation de la vessie au cours du développement) jusqu'aux procédés chirurgicaux ( $p$. ex. lors de valves de l'urètre postérieur chez les garçons).

\section{Mots clés}

Incontinence - Troubles de la miction chez l'enfant · Incontinence nocturne $\cdot$ Appareil de Réveil · Hormone antidiurétique terhose geworfen werden. Ist sie trocken, nass? Stuhlverschmiert? Finden sich anatomische Auffälligkeiten [17]?

Ein einfacher Urinstatus erfolgt zum Ausschluss einer diabetischen Ketoazidose, eines Diabetes insipidus und/oder eines Harnwegsinfektes [17].

Je nach klinischem Kontext sind weitere apparative Untersuchungen indiziert [17]. Eine Uroflowmetrie lässt sich relativ einfach in der Sprechstunde durchführen und gibt Hinweise auf eine Beckenbodendyskoordination oder auf eine subvesi- kale Obstruktion (z.B. posteriore Urethralklappen bei Knaben). Eine Sonographie der Nieren und ableitenden Harnwege erfolgt zum Ausschluss anatomischer Auffälligkeiten, je nach Fragestellung lässt sich auch ein stuhlgefülltes Rektum als Hinweis für eine zugrunde liegende Obstipation darstellen. Im Kindesalter wird die Indikation für eine Schnittbildgebung strenger gestellt, da je nach Alter des Patienten eine Sedierung oder Narkose notwendig ist. Zur Darstellung komplexer anatomischer Verhältnisse (z.B. eines ektopen Ureters) ist das Magnetresonanztomographie jedoch dem Ultraschall überlegen. Weitere diagnostische Untersuchungen wie eine Szintigraphie oder eine Zystomanometrie haben bei diesen Patienten nur in Ausnahmefällen einen Stellenwert.

\section{Management der Enuresis nocturna}

Die Unterscheidung zwischen einer monosymptomatischen und einer nicht- 
Hier steht eine Anzeige.

\section{曾 Springer}




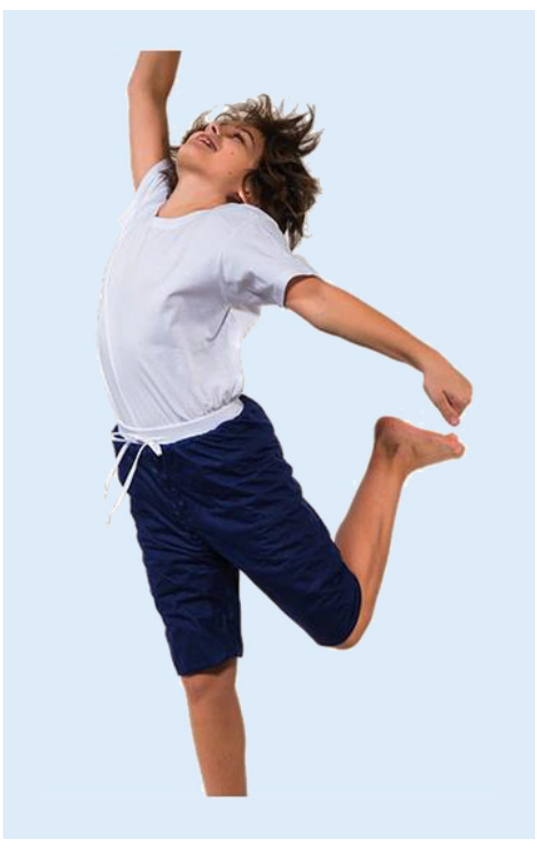

Abb. $2 \triangle$ Beispiel einer aufsaugenden Pyjamahose. (Mit freundlicher Genehmigung der Fa. Urifoon Schweiz. Diese Abbildung fällt nicht unter die Creative Commons CC BY-Lizenz dieser Publikation)

monosymptomatischen Enuresis ist für die Therapiefindung entscheidend. Liegen bei einem Kind Symptome einer Blasenentleerungsstörung, eine sonstige Grunderkrankung oder eine Obstipation vor, sollen diese primär behandelt werden [17]. Sollte danach die Enuresis weiterhin persistieren, wird diese dann in einem zweiten Schritt behandelt.

Beider monosymptomatischen Enuresis ist die Therapiestrategie mit den betroffenen Kindern und Eltern zu evaluieren. Nicht jedes nächtliche Einnässen braucht eine Therapie, wenn das familiäre Umfeld davon nicht stark belastet ist und wenn, v. a. beim Kind, kein Therapiewunsch besteht. In diesen Situationen ist es sicher gerechtfertigt, auf die hohe Spontanheilungsrate zu hoffen.

Zur Grundtherapie gehören sicher eine gute Information über das Krankheitsbild und die Evaluation des Trinkverhaltens. Findet die Flüssigkeitszufuhr mehrheitlich in der zweiten Tageshälfte statt, empfiehlt es sich, die Trinkmenge besser über den Tag zu verteilen. Je nach Alter der Kinder müssen diese aktiv an das Trinken erinnert werden, da im turbulenten Schulalltag kaum Zeit für eine Trinkpause besteht.
Zur Entlastung der Situation im Alltag stehen verschiedene Hilfsmittel zur Verfügung (z.B. Einlagen, aufsaugende Pyjamahosen, Matratzenschoner usw., - Abb. 2), welche teilweise durch die Krankenkasse rückvergütet werden.

Mehrheitlich wird empfohlen, zur besseren Motivation der Kinder nachts keine Windeln/Windelhöschen zu tragen. Wird aber der Wäscheberg zu einer familiären Zerreissprobe, kann es sinnvoll sein, die Windeln/Windelhöschen zu belassen, ebenso dann, wenn das Kind auswärts schläft [18].

Bei kleineren Kindern kann ein Motivationstraining, z.B. mit einem „Sünnelikalender", zum Ziel führen (• Abb. 3; [19]). Jede trockene Nacht wird mit einem „Sünnelikleber“ dokumentiert. Bei einer bestimmten Anzahl erhält das Kind eine Belohnung. Wenn es aber zu viele nasse Nächte gibt, kann das System frustrierend und somit kontraproduktiv sein. Bei grösseren Kindern kann die Motivation nach Erhalt der Belohnung wieder nachlassen und somit keinen langfristigen Erfolg bringen.

Nächtliches Aufnehmen der Kinder durch die Eltern für den Toilettengang wird in aller Regel nicht empfohlen. Einerseits kann die gestörte Nachtruhe zu vermehrter Müdigkeit tagsüber mit allfälligem Leistungsabfall führen, andererseits werden die Kinder nicht konditioniert, die nächtliche Blasenentleerung zu unterdrücken [20].

Je nach Therapieziel der Familie stehen eine medikamentöse Behandlung und/ oder der Einsatz eines Weckapparats zur Verfügung (• Abb.4). Wichtig ist, das betroffene Kind in die Diskussion mit einzubeziehen, da die Therapiewünsche von Eltern und Kind durchaus voneinander abweichen können. Ausschlaggebend für den Erfolg der Therapie ist die Motivation des Kindes.

Die medikamentöse Therapie führt meistens $\mathrm{zu}$ einem schnelleren Erfolg bezüglich trockener Nächte, ist aber in der längerfristigen Therapie dem Weckapparat etwas unterlegen [21]. Jedoch verlangt die Konditionierung mittels Weckapparats eine höhere Compliance der ganzen Familie.

Mittel der Wahl zur medikamentösen Behandlung der monosymptomatischen
Enuresis ist Desmopressin, ein synthetisch hergestelltes Vasopressinanalogon. Die höchste Wirksamkeit hat Desmopressin bei Kindern mit nächtlicher Polyurie [20]. Desmopressin ist gut verträglich und zeigt wenige Nebenwirkungen. Die wichtigste Nebenwirkung ist die Wasserintoxikation bei erhöhter Flüssigkeitszufuhr nach Einnahme des Medikaments. Die Familien sind darauf hinzuweisen, dass ca. $1 \mathrm{~h}$ vor der Einnahme von Desmopressin eine Flüssigkeitsrestriktion stattfinden soll. Das Desmopressin wird ca. $1 \mathrm{~h}$ vor dem Schlafengehen eingenommen. Desmopressin steht in verschiedenen Applikationsformen zur Verfügung, wobei die Schmelztabletten heute Standard sind. Die Startdosis der Schmelztabletten beträgt $120 \mu \mathrm{g}$ und kann bei schlechtem Ansprechen nach 7 Tagen auf die doppelte Dosis gesteigert werden [20]. Die Maximaldosis beträgt $360 \mu \mathrm{g}$. Die Schmelztabletten sind für die Altersgruppe der betroffenen Kinder ein relativ einfacher Applikationsweg und haben eine hohe Akzeptanz. Eine routinemässige Laborkontrolle des Serumnatriumspiegels ist nicht notwendig [22]. Desmopressin in Form von Nasenspray ist nicht mehr für diese Indikation zugelassen, da es mit einem höheren Risiko für hyponatriämische Krampfanfälle verbunden ist [23]. Die Therapie mit Desmopressin kann entweder situativ, z. B. bei externen Übernachtungen, oder über eine längere Periode von 3 Monaten erfolgen. Nach 3 Monaten empfiehlt es sich, die Therapie zu pausieren und den Therapieerfolg zu kontrollieren. Bei wiederkehrender Enuresis können weitere Zyklen von Desmopressin während jeweils 3 Monaten durchgeführt werden. Dabei wird jeweils eine 1- bis 2-wöchige Pause zur Überprüfung des Therapieerfolgs dazwischen empfohlen.

Soll eine langfristige Therapie beendet werden, wird ein Ausschleichen der Dosis innerhalb von 2 Wochen empfohlen. In einer Metaanalyse zeigte dies einen besseren endgültigen Therapieerfolg als ein abruptes Absetzen [24].

Trizyklische Antidepressiva sind eine weitere Therapieoption, die aber sehr restriktiv eingesetzt und erst bei Nichtansprechen sämtlicher anderer Optionen 


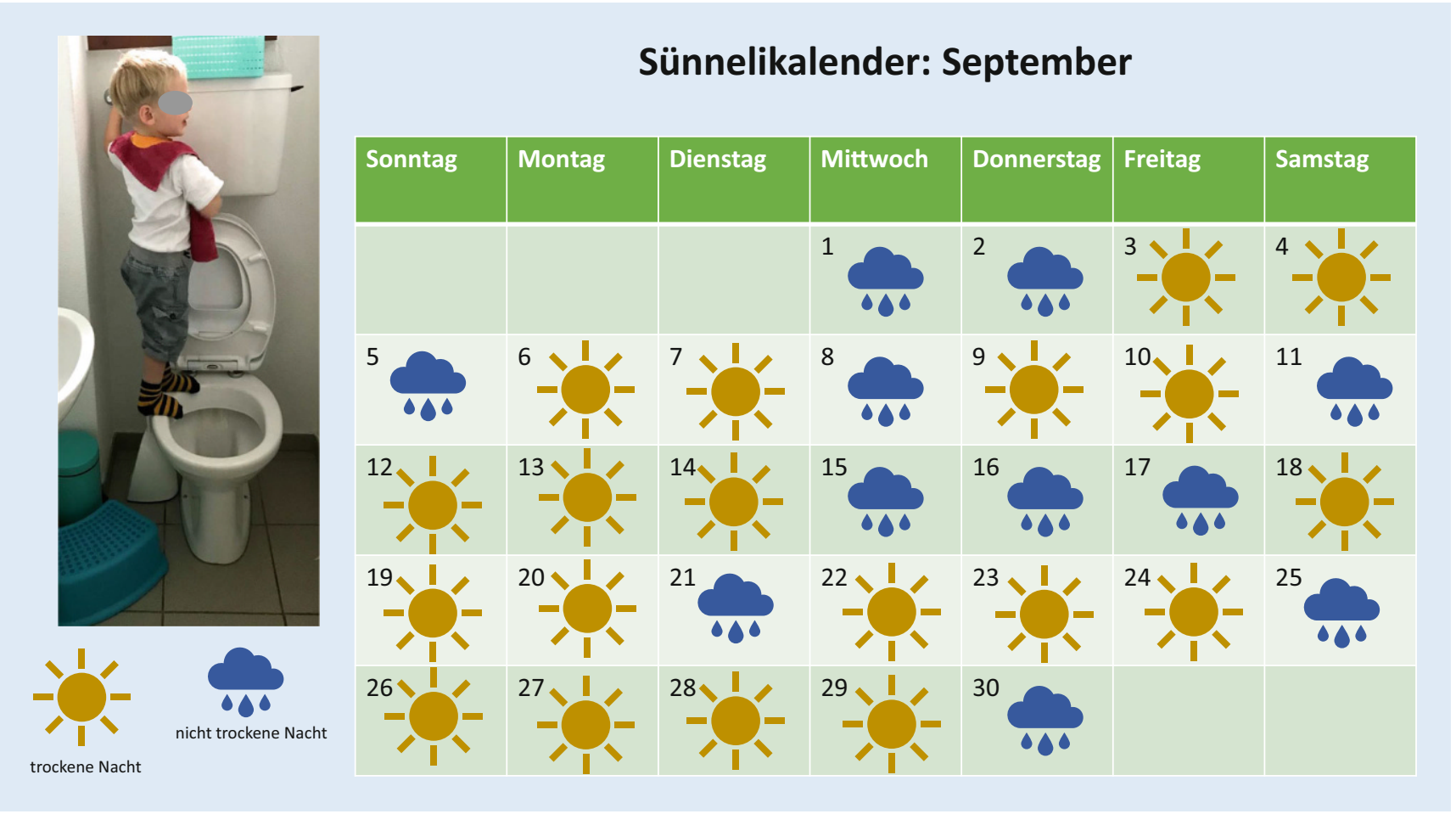

Abb. 3 ॥ Beispiel eines „Sünnelikalenders“. (Foto: privat, kindsgerechte Toilette mit Sitzverkleinerung und Schemel für die Füsse)
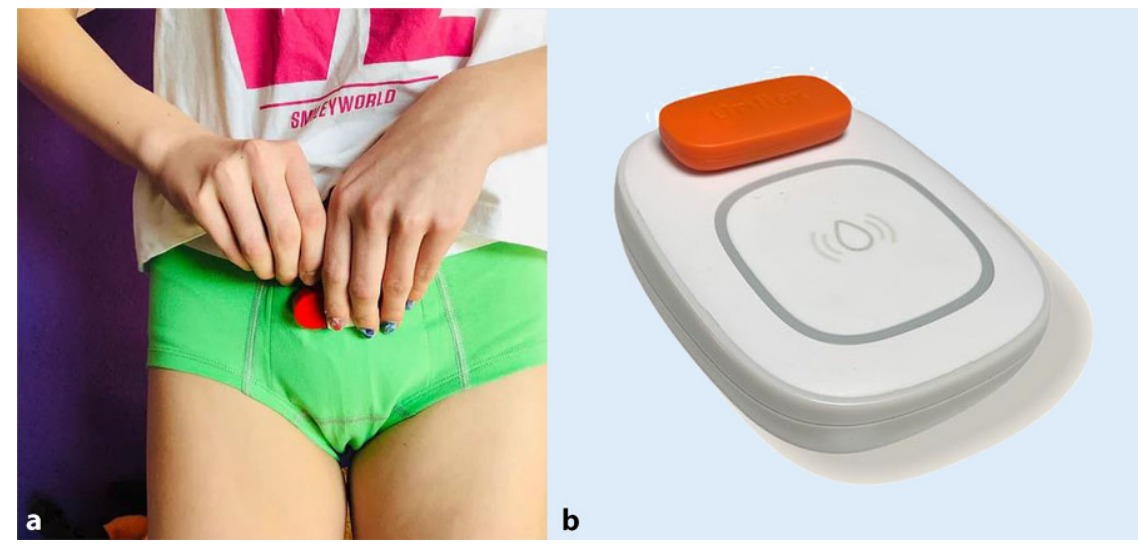

Abb. $4 \Delta$ Beispiel eines kabellosen Weckapparates mit Sensorunterhosen. (Mit freundl. Genehmigung der Fa. Urifoon Schweiz. Diese Abbildung fällt nicht unter die Creative Commons CC BY-Lizenz dieser Publikation)

zum Einsatz kommen sollte. Trizyklische Antidepressiva stimulieren die ADH-Sekretion und wirken detrusorrelaxierend. Das bekannteste Präparat ist Imipramin. In bis zu $5 \%$ der behandelten Kinder kommt es zu erheblichen neurologischen oder kardialen Nebenwirkungen [20].

Der Weckapparat wirkt mittels Konditionierung der Kinder, indem sie lernen, durch den Harndrang aufzuwachen oder die Blasenkontraktion im Schlafzu unterdrücken, bevor es zum Einnässen kommt.
Der Weckapparat besteht aus einem Sensor und einer Alarmeinheit. Heutzutage sind die meisten Geräte kabellos und somit einfacher im Handling. Die Kosten der Miete eines solchen Weckapparats übernimmt teilweise die Krankenkasse. Die Therapie sollte für mindestens $3 \mathrm{Mo-}$ nate am Stück durchgeführt werden, um ein Ansprechen auf die Therapie beurteilen zu können [25]. Dies erfordert häufig eine grosse Compliance der Familie, da die Geräte sehr laut sind. Typischerweise wird berichtet, dass anfänglich durch den Alarm alle Familienmitglieder geweckt werden, nur nicht das betroffene Kind. Sollte sich keine deutliche Verbesserung der Enuresis nach 3-4 Monaten zeigen, empfiehlt es sich, die Therapie zu pausieren und zu einem späteren Zeitpunkt erneut einzusetzen. Je älter das Kind ist, desto höher sind die Erfolgschancen [25].

Sowohl Desmopressin als auch der Weckapparat sind wirksam in der Behandlung der Enuresis nocturna [20]. Hierzu gibt es Metaanalysen, die die Behandlung mit dem Weckapparat vs. Desmopressin vergleichen. Der Erfolg wird etwa als gleichwertig beschrieben, auch wenn die Daten der Metaanalyse von Caldwell et al. [25] etwas limitiert sind. In einer weiteren Metaanalyse von Song et al. [21] scheint der Therapieerfolg mit dem Weckapparat nachhaltiger zu sein und zeigte weniger Therapieversager.

\section{Fazit für die Praxis}

- Abklärungen sind erst ab dem 5. Lebensjahr empfohlen.

- Die Unterscheidung von monosymptomatischer vs. nichtmonosympto- 
matischer Enuresis ist essenziell, da sich die jeweiligen Behandlungsstrategien deutlich unterscheiden.

- Bei der monosymptomatischen Enuresis nocturna sind weiterhin der Weckapparat und/oder die medikamentöse Therapie mit Desmopressin Standard.

- Die Enuresis nocturna zeigt prinzipiell eine hohe Spontanheilungsrate.

\section{Korrespondenzadresse}

\section{Dr. Vivienne Sommer}

Abteilung Kinderchirurgie/Kinderurologie, Universitätskinderspital beider Basel UKBB Spitalstraße 33, 4056 Basel, Schweiz vivienne.sommer-joergensen@ukbb.ch

Funding. Open access funding provided by University of Basel

\section{Einhaltung ethischer Richtlinien}

Interessenkonflikt. V. Sommer, S. Holland-Cunz und $M$. Frech geben an, dass kein Interessenkonflikt besteht.

Für diesen Beitrag wurden von den Autoren keine Studien an Menschen oder Tieren durchgeführt. Für die aufgeführten Studien gelten die jeweils dort angegebenen ethischen Richtlinien.

Open Access. Dieser Artikel wird unter der Creative Commons Namensnennung 4.0 International Lizenz veröffentlicht, welche die Nutzung, Vervielfältigung Bearbeitung, Verbreitung und Wiedergabe in jeglichem Medium und Format erlaubt, sofern Sie den/die ursprünglichen Autor(en) und die Quelle ordnungsgemäß nennen, einen Link zur Creative Commons Lizenz beifügen und angeben, ob Änderungen vorgenommen wurden.

Die in diesem Artikel enthaltenen Bilder und sonstiges Drittmaterial unterliegen ebenfalls der genannten Creative Commons Lizenz, sofern sich aus der Abbildungslegende nichts anderes ergibt. Sofern das betreffende Material nicht unter der genannten Creative Commons Lizenz steht und die betreffende Handlung nicht nach gesetzlichen Vorschriften erlaubt ist, ist für die oben aufgeführten Weiterverwendungen des Materials die Einwilligung des jeweiligen Rechteinhabers einzuholen.

Weitere Details zur Lizenz entnehmen Sie bitte der Lizenzinformation auf http://creativecommons.org/ licenses/by/4.0/deed.de.

\section{Literatur}

1. Austin PF, Bauer SB, Bower W, Chase J, Franco I, Hoebeke P, Rittig S, Walle JV, von Gontard A, Wright A, Yang SS, Nevéus T (2016) The standardization of terminology of lower urinary tract function in children and adolescents: update report from the standardization committee of the International Children's Continence Society. Neurourol Urodyn 35(4):471-481

2. Fergusson DM, Horwood LJ, Shannon FT (1986) Factors related to the age of attainment of nocturnal bladder control: an 8-year longitudinal study. Pediatrics 78(5):884-890

3. Bakker E, van Sprundel M, van der Auwera JC, van Gool JD, Wyndaele JJ (2002) Voiding habits and wetting in a population of 4,332 Belgian schoolchildren aged between 10 and 14 years. Scand J Urol Nephrol 36(5):354-362. https://doi. org/10.1080/003655902320783863

4. Howe AC, Walker CE (1992) Behavioral management of toilet training, enuresis, and encopresis. Pediatr Clin North Am 39(3):413-432. https://doi. org/10.1016/s0031-3955(16)38336-5

5. Klackenberg G (1981) Nocturnal enuresis in a longitudinal perspective. A primary problem of maturity and/or a secondary environmental reaction? Acta Paediatr Scand 70(4):453-457. https://doi.org/10. 1111/j.1651-2227.1981.tb05722.x

6. Forsythe WI, Redmond A (1974) Enuresis and spontaneous cure rate. Study of 1129 enuretis Arch Dis Child 49(4):259-263. https://doi.org/10. 1136/adc.49.4.259

7. Robson WL, Leung AK (2000) Secondary nocturnal enuresis. Clin Pediatr 39(7):379-385. https://doi. org/10.1177/000992280003900701

8. Bloom DA, Seeley WW, Ritchey ML, McGuire EJ (1993) Toilet habits and continence in children: an opportunity sampling in search of normal parameters. J Urol 149(5):1087-1090. https://doi. org/10.1016/s0022-5347(17)36304-8 (Erratum in: JUrol 1993 Dec;150(6):1924)

9. Stadtler AC, Gorski PA, Brazelton TB (1999) Toilet training methods, clinical interventions, and recommendations. American Academy of Pediatrics. Pediatrics 103(6 Pt2):1359-1368

10. Bakwin H (1971) Enuresis in twins. Am J Dis Child 121(3):222-225. https://doi.org/10.1001/ archpedi.1971.02100140088007

11. Bakwin H (1973) The genetics of enuresis. In: Kolvin RM, Meadows SR (Hrsg) Bladder control and enuresis. Medical Books, London, $\mathrm{S} 73$

12. Rittig S, Schaumburg HL, Siggaard C, Schmidt F, Djurhuus JC (2008) The circadian defect in plasma vasopressin and urine output is related to desmopressin response and enuresis status in children with nocturnal enuresis. J Urol 179(6):2389-2395. https://doi.org/10.1016/j.juro. 2008.01.171

13. Van Hoeck K, Bael A, Lax H, Hirche H, Van Dessel E, Van Renthergem D, van Gool JD (2007) Urine output rate and maximum volume voided in school-age children with and without nocturnal enuresis. J Pediatr 151(6):575-580. https://doi. org/10.1016/j.jpeds.2007.05.023

14. Hunsballe JM (2000) Increased delta component in computerized sleep electroencephalographic analysis suggests abnormally deep sleep in primary monosymptomatic nocturnal enuresis. Scand J Urol Nephrol 34(5):294-302. https://doi. org/10.1080/003655900750048305

15. Yeung CK, Diao M, Sreedhar B (2008) Cortical arousal in children with severe enuresis. $\mathrm{N} \mathrm{Engl} \mathrm{J}$ Med 358(22):2414-2415. https://doi.org/10.1056/ NEJMc0706528

16. Bader G, Nevéus T, Kruse S, Sillén U (2002) Sleep of primary enuretic children and controls. Sleep 25(5):579-583

17. Neveus T, Eggert P, Evans J, Macedo A, Rittig $S$, Tekgül S, Vande Walle J, Yeung CK, Robson L,
International Children's Continence Society (2010) Evaluation of and treatmentformonosymptomatic enuresis: a standardization document from the International Children's Continence Society. J Urol 183(2):441-447. https://doi.org/10.1016/j.juro. 2009.10.043

18. Schmitt BD (1997) Nocturnal enuresis. Pediatr Rev 18(6):183-190. https://doi.org/10.1542/pir.18-6183 (quiz 91)

19. Caldwell PH, Nankivell G, Sureshkumar P (2013) Simple behavioural interventions for nocturnal enuresis in children. Cochrane Database Syst Rev. https://doi.org/10.1002/14651858.CD003637. pub3

20. Nevéus T, Fonseca $E$, Franco I, Kawauchi $A$, Kovacevic L, Nieuwhof-LeppinkA, Raes A, TekgülS, Yang SS, Rittig S (2020) Management and treatment of nocturnal enuresis-an updated standardization document from the International Children's Continence Society. J Pediatr Urol 16(1):10-19. https://doi.org/10.1016/j.jpurol. 2019.12.020

21. Song $P$, Huang $C$, Wang $Y$, Wang $Q$, Zhu W, Yue $Y$, Wang W, Feng J, He X, Cui L, Wan T, Wen J (2019) Comparison of desmopressin, alarm, desmopressin plus alarm, and desmopressin plus anticholinergic agents in the management of paediatric monosymptomatic nocturnal enuresis: a network meta-analysis. BJU Int 123(3):388-400. https://doi.org/10.1111/bju.14539

22. National Institute for Health and Care Excellence Nocturnal enuresis - the management of bedwetting in children and young people. www.nice. org.uk/guidance/index.jsp?action=download\& $0=51367$.Zugegriffen: 28. Febr. 2011

23. Food and Drug Administration (2007) Safety alerts for human medical products: desmopressin acetate (marketed as DDAVP nasal spray, DDAVP Rhinal tube, DDAVP, DDVP, Minirin, and Stimate nasal spray). www.fda. gov/Safety/MedWatch/Safetylnformation/ SafetyAlertsforHumanMedicalProducts/ ucm 152113.htm. Zugegriffen:26. Aug. 2011

24. Chua ME, Silangcruz JM, Chang SJ, Williams K, Saunders M, Lopes RI, Farhat WA, Yang SS (2016) Desmopressin withdrawal strategy for pediatric enuresis: a meta-analysis. Pediatrics 138(1):e20160495. https://doi.org/10.1542/peds. 2016-0495

25. Caldwell $\mathrm{PH}$, Codarini $M$, Stewart $F$, Hahn D, Sureshkumar P (2020) Alarm interventions for nocturnal enuresis in children. Cochrane Database Syst Rev. https://doi.org/10.1002/14651858. CD002911.pub3

Hinweis des Verlags. Der Verlag bleibt in Hinblick auf geografische Zuordnungen und Gebietsbezeichnungen in veröffentlichten Karten und Institutsadressen neutral. 